\title{
Thermalization by Elastic Collisions: Positronium in a Rare Gas Moderator
}

\author{
William C. Sauder* \\ Institute for Materials Research, National Bureau of Standards, Washington, D.C. 20234
}

(July 20, 1967)

\begin{abstract}
The energy decay of particles moving through a moderating medium is discussed for the case in which only elastic collisions occur between these incoming particles and the moderator atoms; the random thermal motion of the moderator atoms is taken into account. It is shown that if the cross section is independent of particle velocity the equation for the energy decay emerges in a rather simple form involving the hyperbolic cotangent. Finally, the theoretical development is applied to estimating the thermalization time of positronium in rare gas moderators, and is shown to agree with the limited experimental results presently available.
\end{abstract}

Key Words: Elastic collisions, positronium, thermalization.

\section{Introduction}

In several areas of physics processes occur in which energetic particles enter a moderating medium and subsequently are brought to thermal equilibrium with the atoms of this medium by means of collisions. Examples are found in neutron age theory [1] ${ }^{1}$ and in the literature of positron annihilation in matter [2]. Many previous theoretical treatments have included the approximation that the thermal energy of the moderator atoms can be neglected; obviously, this is not valid if one is interested in the terminal portion of the thermalization process. As will be shown below, it is possible to carry out a simplified treatment of the thermalization problem and yet to take the moderator energy into account.

In the following, slowing of particles solely by means of elastic collisions will be discussed; the thermal motion of the moderating atoms will appear explicitly in the treatment. The resulting representation of the energy decay of the particles as a function of time will involve an integral over the particle velocities; for the special case in which the collision cross section is a constant, the integration will be carried out in closed form to yield a rather succinct equation for the thermalization process. It will be demonstrated in the last section of the paper that results derived herein are useful in considering positronium atoms in rare gas moderators.

\footnotetext{
*Associate Professor of Physics, Virginia Military Institute, Lexington, Va. 24450. Consultant to the Crystal Chemistry Section, Inorganic Materials Division. National Bureau of Standards. Washington, D.C. 20234.

' Figures in brackets indicate the literature references at the end of this paper.
}

\section{Average Energy Loss}

Particles of mass $m$, velocity $\mathbf{v}_{1}$, and momentum $\mathbf{p}_{1}=m \mathbf{v}_{1}$ enter a moderating medium and each collides elastically with a moderator atom of mass $m_{m}$, velocity $\mathbf{v}_{m}$, and momentum $\mathbf{p}_{m}=m_{m} \mathbf{v}_{m}$. It is straightforward to show that the momentum of the incoming particle in the center of mass system is related to these quantities as follows:

$$
\mathbf{p}_{1 c}=\frac{m_{m} \mathbf{p}_{1}-m \mathbf{p}_{m}}{M}=\mathbf{p}_{1}-\frac{m}{M} \mathbf{p}_{c}
$$

where $\mathbf{p}_{1 c}$ is measured in center of mass coordinates, $\mathbf{v}_{c}$ is the velocity of the center of mass, and

$$
\mathbf{p}_{c}=\left(m+m_{m}\right) \mathbf{v}_{c}=M \mathbf{v}_{c} .
$$

If, after the collision, an incoming particle scatters with velocity and momentum $\mathbf{v}_{2}$ and $\mathbf{p}_{2}$ respectively, then it is also true that

$$
\mathbf{p}_{2 c}=\mathbf{p}_{2}-\frac{m}{M} \mathbf{p}_{c}
$$

Subtracting eq (la) from eq (lb) yields the result that the momentum transfer is the same in both coordinate systems:

$$
\mathbf{p}_{2}-\mathbf{p}_{1}=\mathbf{p}_{2 c}-\mathbf{p}_{1 c} .
$$

Using eq (1) one can obtain

$\mathbf{p}_{1} \cdot \mathbf{p}_{2}=\mathbf{p}_{1 c} \cdot \mathbf{p}_{2 c}+\left(\frac{m}{M}\right) \mathbf{p}_{c} \cdot\left(\mathbf{p}_{1 c}+\mathbf{p}_{2 c}\right)+\left(\frac{m}{M}\right)^{2} \mathbf{p}_{c}^{2}$. 
Squaring both sides of eq (2), and employing the fact that momentum and energy conservation in the collision imply that $p_{1 c}=p_{2 c}$, yield the following expression for the change in momentum-squared of the incoming particle:

$$
p_{2}^{2}-p_{1}^{2}=2\left(\frac{m}{M}\right) \mathbf{p}_{c} \cdot\left(\mathbf{p}_{2 c}-\mathbf{p}_{1 c}\right) .
$$

In order to derive an expression for the mean energy loss per collision, oné must average eq (4) (which is equivalent to an expression for the energy loss in any given collision) over all possible angles of incidence between $\mathbf{p}_{1}$ and $\mathbf{p}_{m}$, over all scattering angles, and over the distribution (Maxwellian or otherwise) of moderator velocities. We shall carry out these averages under the assumptions that (1) the moderator motion is completely random, and (2) the scattering is spherically symmetric in the center of mass system. These assumptions can be expressed mathematically as follows:

random moderator: $\quad\left\langle\mathbf{p}_{1} \cdot \mathbf{p}_{m}\right\rangle_{\bmod }=\left\langle\mathbf{p}_{1} \cdot \mathbf{p}_{c}\right\rangle_{\bmod }=0$

isotropic scattering: $\quad\left\langle\mathbf{p}_{1 c} \cdot \mathbf{p}_{2 c}\right\rangle_{\text {scat }}=0$.

Averaging eq (4) overall scattering angles according to eq $(5 b)$ results in

$$
\left\langle\mathbf{p}_{2}^{2}\right\rangle_{\text {scat }}-\mathbf{p}_{1}^{2}=-2\left(\frac{m}{M}\right) \mathbf{p}_{c} \cdot \mathbf{p}_{1 c} .
$$

Since $\mathbf{p}_{c}=\mathbf{p}_{1}+\mathbf{p}_{m}$, then performing the averaging of eq (6) over moderator velocities [eq (5a)] yields the following expression for the fractional energy change:

$$
\frac{\left\langle\left\langle v_{2}^{2}\right\rangle_{\text {scat }}\right\rangle_{\bmod }-v_{1}^{2}}{v_{1}^{2}}=-2\left(1+m / m_{m}\right)^{-2}\left\{\left(\frac{m}{m_{m}}\right)-\frac{\left\langle v_{m}^{2}\right\rangle}{v_{1}^{2}}\right\}
$$

where $\left\langle v_{m}^{2}\right\rangle$ is the mean-squared moderator velocity. Having obtained this expression for mean energy loss, we will (for convenience) drop the average bracket notation in all further work.

The effect of taking the thermal energy of the moderator into account can be identified with the term $\left(v_{m} / v_{1}\right)^{2}$. As the velocity of the incoming particle is reduced through elastic collisions to a magnitude comparable to that of the moderator atoms, the energy lost in successive collisions becomes smaller. Finally, when the particle reaches thermal equilibrium, that is, when

$$
\frac{1}{2} m v_{1}^{2}=\frac{1}{2} m_{m} v_{m}^{2},
$$

then no further decrease in energy occurs.

\section{Thermalization}

Usually for the physical situations mentioned in the introductory paragraphs the left hand member of eq (7) is sufficiently small in magnitude so that one may write

$$
\left(v_{2}^{2}-v_{1}^{2}\right) / v_{1}^{2} \simeq d\left(\ln v^{2}\right) .
$$

Thus eq (7) can be written in differential form,

$$
d(\ln v)=-\left(1+m / m_{m}\right)^{-2}\left\{\left(\frac{m}{m_{m}}\right)-\left(\frac{v_{m}}{v}\right)^{2}\right\}
$$

which describes a continuous process rather than the discreet process indicated in (7).

We now take from kinetic theory [3] the result that the mean collision rate of the incoming particles with the moderator is

$$
\nu=\rho_{n} \sigma v
$$

where $\sigma=$ the elastic collision cross section and $\rho_{n}=$ the number of moderator atoms per $\mathrm{cm}^{3}$. Combining (9) and (10) leads to the differential equation governing the thermalization process:

$$
\frac{d(\ln v)}{d t}=-\frac{\rho_{n} \sigma v}{\left(1+R_{m}\right)^{2}}\left\{R_{m}-\left(\frac{v_{m}}{v}\right)^{2}\right\}
$$

where

$$
R_{m}=m / m_{m}
$$

Separation of variables leads to the following integral:

$$
\int_{v_{0}}^{v} \frac{d v}{\sigma(v)\left[R_{m} v^{2}-v_{m}^{2}\right]}=-\frac{\rho_{n} t}{\left(1+R_{m}\right)^{2}}
$$

where $v_{0}$ is the initial velocity of the incoming particle as it enters the moderating medium.

Equation (12) cannot be integrated unless the explicit dependence of the cross section upon velocity is known. For the case in which $\sigma$ is a constant the results are particularly concise. If the quantities $\alpha$ and $\beta$ are defined as follows:

$$
\begin{aligned}
& \alpha=\frac{R_{m}^{1 / 2} \rho_{n} \sigma v_{m}}{\left(1+R_{m}\right)^{2}} \\
& \beta=\operatorname{coth}^{-1} \sqrt{\frac{E_{0}}{E_{m}}}
\end{aligned}
$$

where $E_{0}$ and $E_{m}$ are the kinetic energies corresponding to $v_{0}$ and $v_{m}$, respectively, then the integration of eq (12) for $\sigma$ constant yields

$$
E / E_{m}=\operatorname{coth}^{2}(\beta+\alpha t)
$$

where $E$ is the kinetic energy of the incoming particle. Note that $\alpha$ can be related to the collision frequency of the incoming particle at thermal equilibrium by employing eq (8):

$$
\alpha=\frac{m m_{m}}{\left(m+m_{m}\right)^{2}} \rho_{n} \sigma v_{T}=\frac{m m_{m}}{\left(m+m_{m}\right)^{2}} \nu_{T}
$$


where $v_{T}$ and $\nu_{T}$ are the velocity and the collision frequency of the thermalized particle, respectively.

The form of the thermalization equation, eq (15), is demonstrated in figure 1 in which is plotted the function $\operatorname{coth}^{2}(\alpha t)$. One can see from eq (15) that $\beta$ indicates the point of the curve at which the thermalization process is initiated. Note that when $\beta$ is small, for all intents and purposes thermal equilibrium is established after the characteristic time interval $\alpha^{-1}$.

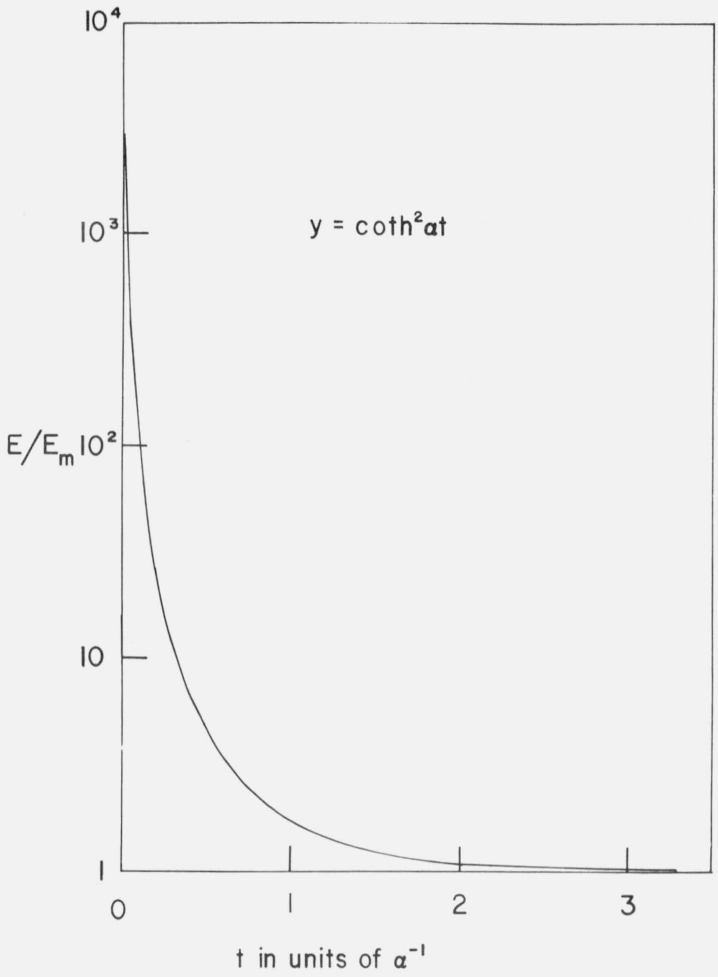

FIGURE 1. The energy decay function for velocity-independent cross section.

\section{Thermalization of Positronium}

When positrons enter matter, positronium atoms are formed only after the kinetic energy of the positrons has fallen to a value lying in the so-called Ore gap [4]; this region lies below the ionization potential of the moderator atoms, and typically is several electron volts wide; therefore the Ore gap occupies the region $1-20 \mathrm{eV}$. If the moderator is composed of rare gas atoms, then the elastic collision is the only energy loss mechanism available to the positronium. Massey and Mohr [5] indicate that the elastic collision cross section for positronium in the Ore gap is of the order of magnitude of the geometrical cross section, and that furthermore, this cross section increases at lower energies.
Equation (15) of the preceeding section is suitable for making conservative estimates of the energy of positronium at the time of its annihilation if one uses for $\sigma$ the geometrical cross section, which, as stated above, is a lower limit for the true elastic collision cross section. Such estimates are of interest since the energy of the annihilating positronium atom is related to the width of the angular distribution of the photons emitted in the annihilation process.

Consider the thermalization of positronium in argon gas at $300{ }^{\circ} \mathrm{K}$ and $2 \mathrm{~atm}$ pressure. Using for the radius of the argon atom a value of $1.5 \AA$ in order to determine the geometrical cross section, and making use of the fact that argon under the stated conditions behaves approximately as an ideal gas, we have computed the value of $\alpha$ to be

$$
\alpha=3.9 \times 10^{6} \sec ^{-1} \text { (Ps in argon). }
$$

Thus the estimated thermalization time for Ps in argon is $\alpha^{-1}=2.6 \times 10^{-7} \mathrm{sec}$. Since the Ore gap for argon lies between 9.0 and $11.6 \mathrm{eV}$, then the ratio $E_{0} / E_{m}$ is approximately 100 .

The only existing measurements of angular distributions in a rare gas that are of sufficient accuracy to test this result were performed by Heinberg and Page [6]. In that experiment, argon at room temperature and $2 \mathrm{~atm}$ pressure was employed as a moderating medium; the experimental sensitivity was such that the authors were able only to state that the time required for the positronium kinetic energy to fall to $0.5 \mathrm{eV}$ is greater than $9 \times 10^{-9} \mathrm{sec}$.

Using the values of $\alpha$ and $E_{0} / E_{m}$ determined above, eq (15) indicates that the time for the Ps energy to reach $0.5 \mathrm{eV}$ is $5 \times 10^{-8} \mathrm{sec}$, which agrees nicely with the experimental results.

I thank R. D. Deslattes of the National Bureau of Standards for many helpful discussions during the time that the above results were being derived. I am particularly grateful to R. C. Casella of the National Bureau of Standards for the derivation of the energy loss equation [eq. (7)] in terms of expressions in momentum space; this is far more elegant than the geometrical derivation that had been done originally.

\section{References}

[1] Samuel Glasstone and Milton C. Edlund, The Elements of Nuclear Reactor Theory, chp. VI (D. Van Nostrand Co., Inc., New York, 1952).

[2] P. R. Wallace, Phys. Rev. 100, 738 (1955).

[3] E. H. Kennard, Kinetic Theory of Gases, p. 104 (McGraw-Hill Book Co., Inc., New York, 1938).

[4] M. Deutsch, Prog. Nuc. Phys. 3, 131 (1953).

[5] H. S. W. Massey and C. B. O. Mohr, Proc. Phys. Soc. (London) A6 7, 695 (1954).

[6] Milton Heinberg and Lorne A. Page, Phys. Rev. 107, 1589 (1957). 